\title{
HERV-K and HERV-H Env Proteins Induce a Humoral Response in Prostate Cancer Patients
}

\author{
Maria Antonietta Manca ${ }^{1}$, Tatiana Solinas ${ }^{2,3}$, Elena Rita Simula ${ }^{1}{ }^{\oplus}$, Marta Noli $^{1}{ }^{1}$, Stefano Ruberto ${ }^{1}{ }^{(}$, \\ Massimo Madonia ${ }^{2,3}$ and Leonardo A. Sechi ${ }^{1,4, *(D)}$
}

check for

updates

Citation: Manca, M.A.; Solinas, T.; Simula, E.R.; Noli, M.; Ruberto, S.; Madonia, M.; Sechi, L.A. HERV-K and HERV-H Env Proteins Induce a Humoral Response in Prostate Cancer Patients. Pathogens 2022, 11, 95. https://doi.org/10.3390/ pathogens11010095

Academic Editor: Robbie B Mailliard

Received: 7 December 2021 Accepted: 13 January 2022 Published: 14 January 2022

Publisher's Note: MDPI stays neutral with regard to jurisdictional claims in published maps and institutional affiliations.

Copyright: (C) 2022 by the authors. Licensee MDPI, Basel, Switzerland. This article is an open access article distributed under the terms and conditions of the Creative Commons Attribution (CC BY) license (https:// creativecommons.org/licenses/by/ $4.0 /)$.
1 Dipartimento di Scienze Biomediche, Università di Sassari, 07100 Sassari, Italy; m.anto.manca@gmail.com (M.A.M.); simulaelena@gmail.com (E.R.S.); martanoli@outlook.it (M.N.); ruberto.ste@gmail.com (S.R.)

2 Dipartimento di Scienze Mediche, Chirurgiche e Sperimentali, Università di Sassari, 07100 Sassari, Italy; tatiana.solinas@tiscali.it (T.S.); madonia@uniss.it (M.M.)

3 Struttura Complessa di Urologia, Azienda Ospedaliera Universitaria, 07100 Sassari, Italy

4 Struttura Complessa di Microbiologia e Virologia, Azienda Ospedaliera Universitaria, 07100 Sassari, Italy

* Correspondence: sechila@uniss.it

\begin{abstract}
A higher expression of human endogenous retroviruses (HERVs) has been associated with several malignancies, including prostate cancer, implying a possible use as a diagnostic or prognostic cancer biomarker. For this reason, we examined the humoral response against different

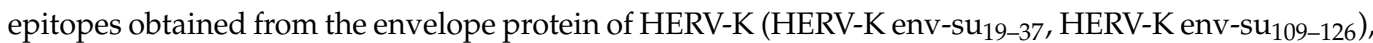
HERV-H (HERV-H env-su 229-241, HERV-H env 387-399) and HERV-W (HERV-W env-su93-108, HERV-W env-su $248-262$ ) in the plasma of patients affected by prostate cancer (PCa), and compared to that of benign prostate hyperplasia (BPH) and a borderline group of patients with atypical small acinar proliferation (ASAP) and prostate intraepithelial neoplasia (PIN) and healthy controls. A significant antibody response was observed against HERV-K env-su ${ }_{109-126}(p=0.004)$ and HERV-H env-su $229-241$ $(p<0.0001)$ in PCa patients compared to HCs, BPH and borderline cohorts, whilst no significance difference was found in the antibodies against HERV-W env-su $93-108$ and HERV-W env-su $248-262$ in patients with PCa. Our results provided further proof of the association between HERV-K and PCa and added new evidence about the possible involvement of HERV-H in PCa pathogenesis, highlighting their possibility of being used as biomarkers of the disease.
\end{abstract}

Keywords: prostate cancer; HERV-H; HERV-K; humoral response; ELISA

\section{Introduction}

Prostate cancer (PCa) is the most common cause of death by cancer in the male population. The incidence rates appear to change depending on race, ethnicity, and geography, but also aging, family history, obesity, and diet [1-3]. Several studies pointed out both chronic inflammation and genitourinary infections as possible contributors in prostatic carcinogenesis and tumor progression [4,5]. PCa is a heterogenous disease, and one of the challenges is the inability of the current diagnostic tests, including the protease-specific antigen (PSA) screening and histopathological grading system, to distinguish between indolent and aggressive tumors [6]. The human genome accounts for approximately $8 \%$ of sequences derived from endogenous retroviruses (ERVs). To date, these sequences are remnants of exogenous retroviral infections of the germ cell line which guaranteed their vertical transmission in a Mendelian fashion [7]. HERVs are not infectious, and their pathogenicity has been dampened due to the accumulation of deleterious mutations, deletions, and epigenetic modifications, such as DNA methylation, which contributed to the repression of HERV expression [8]. However, evidence confirms that, in specific circumstances, HERVs sequences undergo a process of reactivation. The role of some HERV envelope (env) proteins is well known, both in health tissue and 
disease [9-11]. Different mechanisms have been proposed about the role of HERVs in cancer and in the transformation of benign cells. First, HERVs possess 2 long terminal repeats (LTRs), since they share a typical retroviral genomic structure $\left(5^{\prime}\right.$ LTR-gag, pro, pol, env-3'LTR), which can alter the expression of host cell genes, leading to uncontrolled cell proliferation. Second, some HERVs, such as HERV-K, encode proteins with oncogenic properties. HERV-K(HML2) has been recognized as the most recently acquired and active member of the HERVs family, therefore, it retained complete open reading frames (ORFs) and the capability to encode functional proteins [12,13]. HERV-K(HML-2) env gene is characterized by the presence or absence of a $292 \mathrm{bp}$ deletion, which leads to the expression of two splicing variants, Np9 and Rec, respectively [14,15]. Np9 plays an intriguing role in the co-activation of $\beta$-catenin, ERK, Akt and Notch1 promoting the growth of human leukemia stem/progenitor cells [16]. Both Np9 and Rec have been shown to interact, physically and functionally, with the promyelocytic zinc finger (PLZF) tumor suppressor, and inhibit its role as a transcriptional repressor, leading to c-Myc overexpression and alterations in the expression patterns of c-Myc target genes [17]. Despite HERV-K env gene expression in healthy tissues $[18,19]$, its reactivation has been documented in several malignancies, such as melanoma, leukemia, breast cancer, lymphoma, and teratocarcinoma [20-24]. Recently, Sgarbi Reis et al. [25] reported the increased expression of HERV-K gag in PCa tissues, and increased levels of HERV-K gag autoantibodies in a subset of patients with advanced PCa compared to healthy controls (HCs). An increase in HERV-K gag mRNA expression was also found in the peripheral blood mononuclear cells (PBMC) of older men and smokers with PCa [26]. With regard to the epigenetic changes during tumorigenesis, several studies reported an alteration in the expression of both HERV-W and HERV-H, and a specific immune response directed against the two retroviruses [27-29]. The aim of this study was to evaluate the presence of autoantibodies against different epitopes derived from the envelope protein of HERV-K, HERV-W and HERV-H in the plasma of patients with PCa compared to healthy controls (HCs), a cohort of patients with benign prostate hyperplasia (BPH), and a borderline group of patients with atypical small acinar proliferation (ASAP) and prostate intraepithelial neoplasia (PIN).

\section{Results}

The detection of antibodies against the selected highly immunogenic peptides derived from different portions of the envelope protein of HERV-K, HERV-W and HERV-H was carried out by an indirect ELISA assay within the HCs, PCa, BPH and the borderline cohorts. Fifteen out of $105(14 \%)$ patients with PCa $(p=0.004), 6$ out of $74(8 \%)$ patients with $\mathrm{PBH}, 2$ out of $31(6 \%)$ patients of the borderline group, and 6 out of $104(6 \%)$ of the healthy controls were seropositive against HERV-K env-su (Figure 1D). Twenty-eight out of $105(26 \%)$ patients with PCa $(p<0.0001), 16$ out of $74(15 \%)$ patients with $\mathrm{PBH}(p=0.008)-8$ out of $31(25 \%)$ patients of the borderline group and 15 out of $104(14 \%)$ of healthy controls-were seropositive against HERV-H env-su $229-241$ (Figure 1C). No statistically significant difference in the humoral response

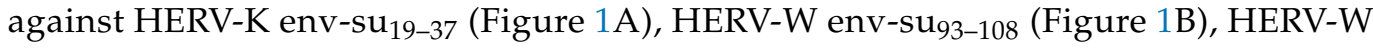
env-su ${ }_{248-262}$ (Figure 1E) and HERV-H env $387-399$ (Figure 1F) has been found between PCa patients and HCs, as well as between PCa and the other disease groups, BPH and borderline. 

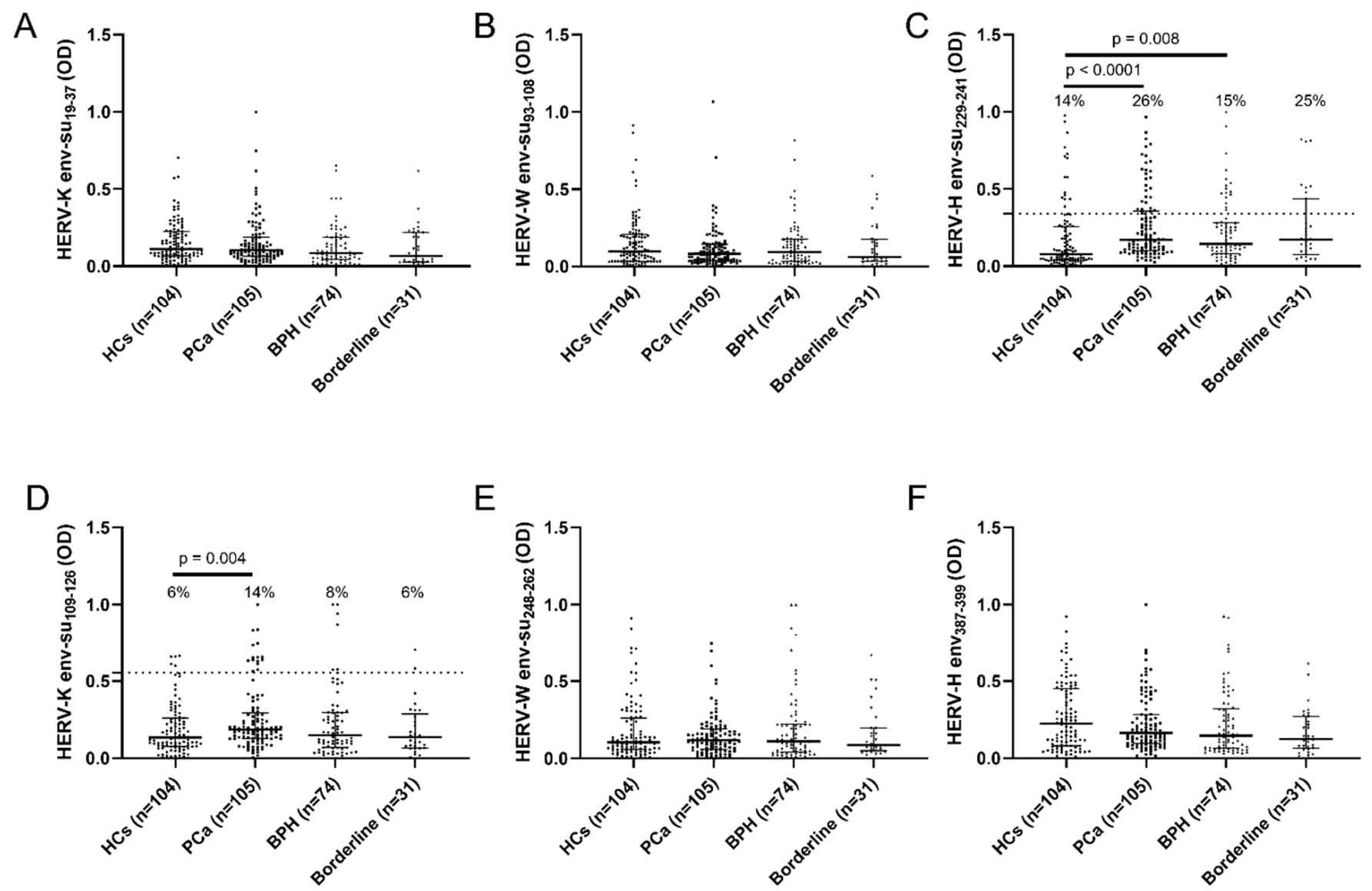

Figure 1. Indirect ELISA assay to detect antibodies against HERV env-derived peptides. Plasma samples from $\mathrm{HCs}, \mathrm{PCa}, \mathrm{BPH}$ and borderline groups were tested to detect circulating antibodies against HERV-K env-su $u_{19-37}$ (A), HERV-K env-su ${ }_{109-126}$ (D), HERV-W env-su93-108 (B), HERV-W env-su 248-262 (E), HERV-H env-su $229-241$ (C) and HERV-H env $387-399$ (F). The black bars represent median plus interquartile range, whether the dotted lines represent the cut-off values for seropositivity obtained after ROC analysis.

To determine whether the humoral response against HERV env-derived peptides was influenced by the patient grading system and the cancer progression, we stratified the population of PCa patients according to the Gleason grading system. The analysis exhibited a statistically significant difference in the antibody response against HERV-K env-su $\mathrm{s}_{109-126}$ (Figure 2D) between HCs and the GS $\geq 8$ group ( $p=0.03$ ). A significant difference was also found in the antibody response directed against HERV-H env-su 229-241 (Figure 2F) between HCs and patients with GS $=6(p=0.001), \mathrm{GS}=7(p=0.03)$ and GS $\geq 8(p=0.01)$. However, no differences were found in the antibody levels among the various GS classes against HERV-H env-su $229-241$, indicating that the immune response directed against the envelope protein of HERV-H was not affected by the Gleason grading and the level of differentiation of prostate tissue. No differences were found in the humoral response against HERV-K env-su ${ }_{19-37}$ (Figure 2A), HERV-W env-su ${ }_{93-108}$ (Figure 2B), HERV-W env-su ${ }_{248-262}$ (Figure 2E) and HERV-H env387-399 (Figure 2F). 
A

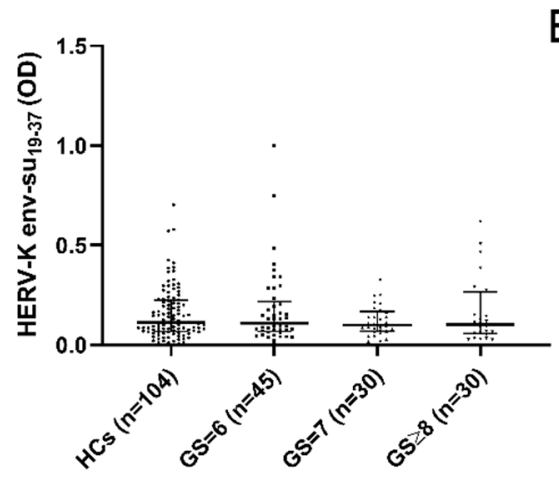

D

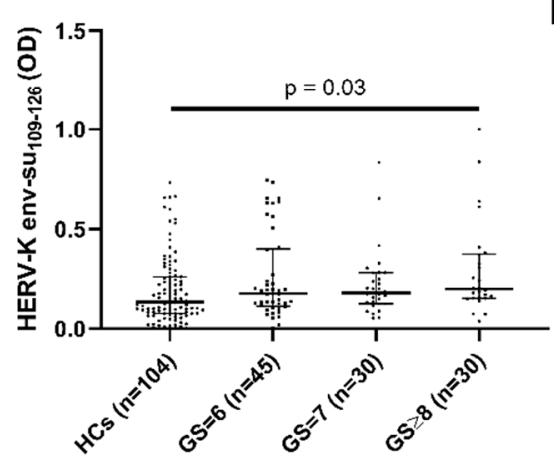

B

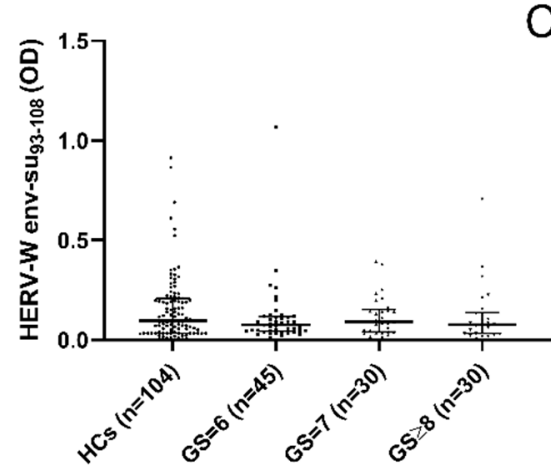

$E$

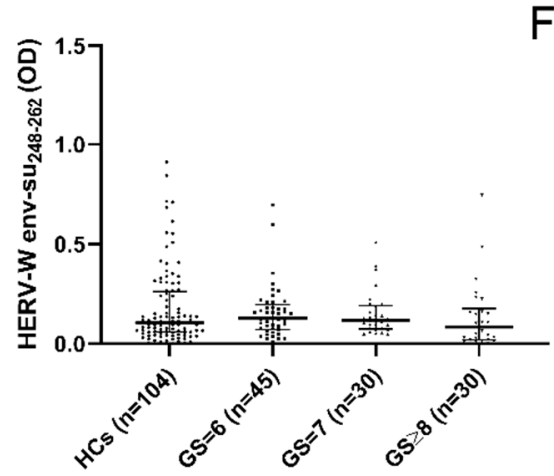

C

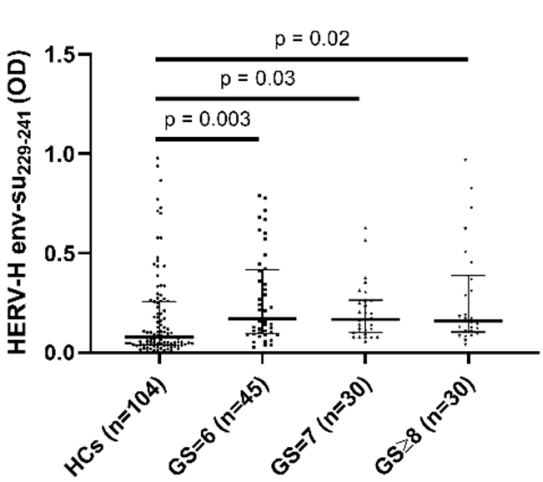

$\mathrm{F}$

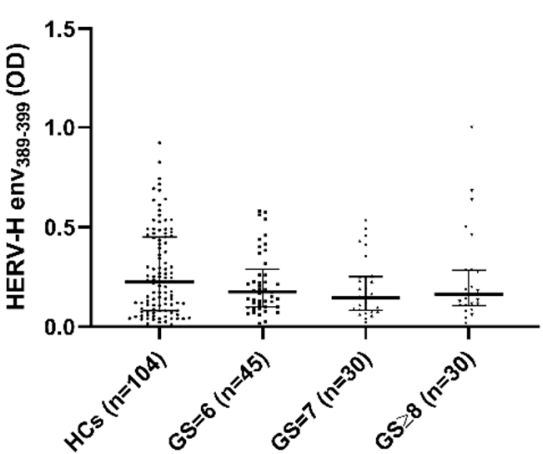

Figure 2. Stratification of PCa patients based on Gleason grading system and analysis of antibody response against HERV-K env-su 19-37 (A), HERV-K env-su ${ }_{109-126}$ (D), HERV-W env-su ${ }_{93-108}$ (B), HERV-W env-su $248-262$ (E), HERV-H env-su $229-241$ (C) and HERV-H env $387-399$ (F). The black bars represent median plus interquartile range.

In Figure 3, Spearman's correlation shows that PSA plasmatic levels are not correlated to the humoral response against HERV env-derived peptides; not only in patients with PCa (Figure 3A), but also in patients with PBH (Figure 3B), and in patients of the borderline group (Figure 3C). In PCa patients (Figure 3A), the results show the following correlations between HERV-K env-su ${ }_{19-37}$ and HERV-K env-su ${ }_{109-126}(r=0.34$, $p=0.0004)$, HERV-W env-su ${ }_{93-108}(\mathrm{r}=0.31, p=0.001)$, HERV-W env-su $248-262(\mathrm{r}=0.34$, $p=0.0004)$, HERV-H env-su $229-241(\mathrm{r}=0.33, p=0.0005)$ and HERV-H env $387-399(\mathrm{r}=0.23$, $p=0.01$ ); low correlations were found between HERV-K env-su $109-126$ and HERV-W env$\mathrm{su}_{93-108}(\mathrm{r}=0.07, p=\mathrm{ns})$, HERV-W env-su $248-262(\mathrm{r}=0.24, p=0.01)$, HERV-H env-su $229-241$ $(\mathrm{r}=0.16, p=\mathrm{ns})$ and HERV-H env $387-399(\mathrm{r}=-0.003, p=\mathrm{ns})$; low correlations were also found between HERV-W env-su ${ }_{93-108}$ and HERV-W env-su $248-262(r=0.39, p<0.0001)$, HERV-H env-su $229-241(\mathrm{r}=0.41, p<0.0001)$, HERV-H env $387-399(\mathrm{r}=0.46, p<0.0001)$ and between HERV-W env-su $248-262$ and HERV-H env-su $229-241(\mathrm{r}=0.37, p=0.0001), \mathrm{HERV}-\mathrm{H}$ $\operatorname{env}_{387-399}(\mathrm{r}=0.21, p=0.02)$. An $\mathrm{r}$ correlation coefficient of 0.47 was found between HERV-H env-su $229-241$ HERV-H env $387-399(p<0.0001$ ). In BPH patients (Figure 3B), we found a correlation between HERV-K env-su ${ }_{19-37}$ and HERV-K env-su ${ }_{109-126}(\mathrm{r}=0.50$, $p<0.0001)$, HERV-W env-su ${ }_{93-108}(\mathrm{r}=0.54, p<0.0001)$, HERV-W env-su $248-262(\mathrm{r}=0.35$, $p=0.002)$, HERV-H env-su $229-241(\mathrm{r}=0.49, p<0.0001)$ and HERV-H env $387-399(\mathrm{r}=0.28$, $p=0.01)$. Low correlations were found between HERV-K env-su $\mathbf{s u}_{109-126}$ and HERV-W env-su 93-108 $(\mathrm{r}=0.37, p=0.001)$, HERV-W env-su $248-262(\mathrm{r}=0.28, p=0.01)$, HERV-H env-su 229-241 $(\mathrm{r}=0.26, p=0.02)$; no significant correlation was found between HERV-K env-su $\mathrm{su}_{10-126}$ and HERV-H env $387-399(\mathrm{r}=0.14, p=\mathrm{ns})$. A moderate correlation was found between HERV-W env-su ${ }_{93-108}$ and HERV-W env-su $248-262(\mathrm{r}=0.45, p<0.0001)$, HERV-H env-su ${ }_{229-241}(\mathrm{r}=0.67, p<0.0001)$, HERV-H env $387-399(\mathrm{r}=0.57, p<0.0001)$. HERV-W 
env-su ${ }_{248-262}$ showed the following correlations with HERV-H env-su $229-241(\mathrm{r}=0.40$, $p<0.0001)$, HERV-H env $387-399(\mathrm{r}=0.22, p=\mathrm{ns})$; whist, a moderate correlation was found between HERV-H env-su $229-241$ and HERV-H env $387-399(r=0.59, p<0.0001)$. In the Borderline group (Figure 3C), a high correlation was found between HERV-K env-su $\mathrm{s}_{19-37}$ and HERV-K env-su ${ }_{109-126}(\mathrm{r}=0.80, p<0.0001)$, HERV-W env-su $248-262(\mathrm{r}=0.73, p<0.0001)$, HERV-H env-su $229-241(\mathrm{r}=0.76, p<0.0001)$, and between HERV-W env-su ${ }_{93-108}$ and HERV$\mathrm{W}$ env-su $248-262(\mathrm{r}=0.77, p<0.0001)$, HERV-H env-su $229-241(\mathrm{r}=0.70, p<0.0001)$. A high correlation was also found between HERV-H env-su $229-241$ and HERV-W env-su $248-262$ $(\mathrm{r}=0.72, p<0.0001)$. A moderate correlation was observed between HERV-K env-su $\mathrm{su}_{109-126}$ and HERV-W env-su s3-108 $(\mathrm{r}=0.47, p=0.007)$, HERV-W env-su $248-262(\mathrm{r}=0.50, p=0.004)$, HERV-H env ${ }_{387-399}(r=0.39, p=0.02)$, between HERV-H env $387-399$ and HERV-W env$\mathrm{su}_{248-262}(\mathrm{r}=0.43, p=0.01)$, HERV-K env-su ${ }_{19-37}(\mathrm{r}=0.49, p=0.005)$, HERV-W env-su ${ }_{93-108}$ $(\mathrm{r}=0.56, p=0.001)$, HERV-H env-su $229-241(\mathrm{r}=0.58, p=0.007)$, and between HERV-K env-su $\mathbf{s}_{19-37}$ and HERV-W env-su's-108 $(\mathrm{r}=0.65, p=0.0001)$.
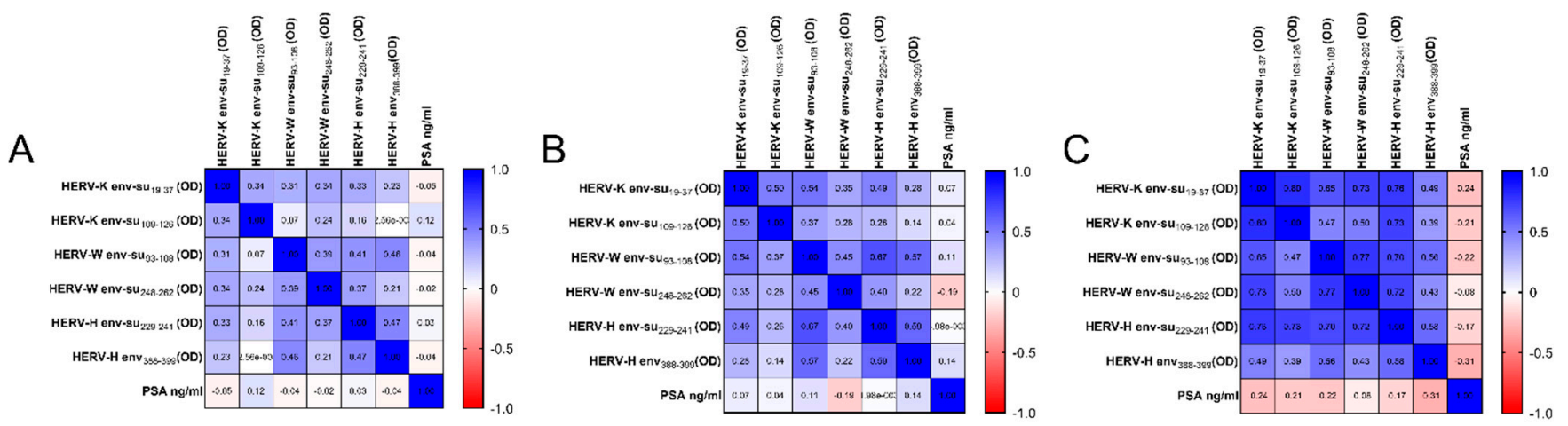

Figure 3. The heatmap shows the Spearman's correlation coefficients among HERV env-derived peptides and PSA plasmatic levels in PCa (A), BPH (B) and borderline (C) cohorts.

\section{Discussion}

Over the years, several studies have investigated the possible involvement of HERV in different diseases, such as multiple sclerosis [30], amyotrophic lateral sclerosis [31], autoimmune diseases [9,32], and especially cancer [33-36]. The envelope protein of HERV family viruses is well known for its immunosuppressive properties and its role in the modulating transcription factors of cancer-associated pathways [16,37]. Regarding PCa, screening methods for diagnosis and prognosis are necessary for cancer management, and to date, healthcare has been using both invasive (biopsy) and low invasive (PSA screening) approaches in PCa diagnoses. Several studies used different approaches to investigate the role played by HERVs in cancer pathogenesis. Recently, Rezaei et al. reported elevated levels of HERV-K gag RNA and protein in malignant regions of the male prostate with PCa compared to matched benign regions [38]. Meanwhile, previous studies reported the presence of serum antibody against HERV-K gag in prostate cancer [25] and an increased expression of HERV-K env in the PBMCs of PCa patients, which appeared to be affected by age and smoking status [26]. Chie et al. instead reported the involvement of HERV$\mathrm{H}$ in cancer immune evasion and its role in amplifying the epithelial-to-mesenchymal transition [39]. This latter study proved the ability of an HERV-H-derived peptide to increase the production of CCL19, and its role as a chemo attractive factor in recruiting an elevated number of immunosuppressive immune cells in HERV-H ${ }^{+}$CCL19 ${ }^{+}$colon cancer tissues. In their work, Pérot et al. suggest a functional role for HERV-H in colorectal carcinogenesis, proving a correlation between HERV-H reactivation and clinical parameters, such as the presence of tumor cells in lymph nodes [40]. Here, we investigated the presence of a humoral response against highly immunogenic peptides derived from the envelope proteins of HERV-K, HERV-W, and HERV-H in the plasma of patients with PCa, BPH and a borderline cohort, given the easy accessibility of such body fluid and its advantage in 
using a specific HERV-directed immune response as a possible disease biomarker. We found that $14 \%$ of patients with PCa displayed higher antibody levels against HERV-K env-su ${ }_{109-126}$ compared to HCs, BPH and the borderline cohorts, while no differences were found in the humoral response against the peptides derived from the envelope protein of HERV-W (HERV-W en-su ${ }_{93-108}$ and HERV-W env-su $248-262$ ). We also found, for the first time, that $26 \%$ of patients with PCa displayed a strong antibody response against $\mathrm{HERV}-\mathrm{H}$ env-su $229-241$ compared to $\mathrm{HCs}, \mathrm{BPH}$, and the borderline cohorts. In the BPH group, $14 \%$ of patients were found seropositive to HERV-H env-su $229-241$, though this result was not confirmed after a T-Fisher exact test. To deepen the significance of HERV-env-specific humoral response, we decided to stratify the population of PCa patients according to the Gleason score system, a powerful predictor of PCa prognosis. Our results pointed out the fact that the humoral response directed against HERV-H env-su $229-241$ did not appear to be correlated to the Gleason grading system and the tumor progression, since no significant differences were found among the GS-based groups, whilst the humoral response against HERV-K env-su ${ }_{109-126}$ appeared stronger in patients with a GS $\geq 8$. These findings support the hypothesis of a potential role for HERV-K env and HERV-H env autoantibodies as prostate cancer biomarkers; it may be useful to help elucidate the diagnostic and prognostic value of HERV serum autoantibodies to further investigate the expression levels of the envelope protein of HERV-K and HERV-H in biopsy samples, as well as PBMC.

\section{Materials and Methods}

\subsection{Study Population and Blood Collection}

This study was approved by the Ethical Committee of AOU Sassari, and all patients signed an informed consent form. The study population consisted of 105 patients with $\mathrm{PCa}, 74$ patients with $\mathrm{BPH}$, and a borderline group of 31 patients diagnosed with ASAP and PIN. The population of HCs consisted of 105 individuals matched by age. The PCa, $\mathrm{BPH}$ and borderline cohorts were recruited from the Urology Department of University Hospital, Sassari, while the HCs were recruited from the Transfusion Center, AOU, Sassari. Peripheral venous blood was collected at the time of the subject recruitment, and was collected in $\mathrm{K}^{+}$-EDTA test tubes, both for patients and HCs. The plasma samples were isolated from whole blood by standard Ficoll-Histopaque (Sigma-Aldrich, St. Louis, MO, USA) gradient centrifugation and stored at $-20^{\circ} \mathrm{C}$; thereafter, they were used to test the presence of autoantibodies against HERV env-derived peptides by an indirect ELISA assay. Clinical data about the patients and HCs are shown in Table 1.

Table 1. Demographic and clinical characteristics of patients and healthy controls.

\begin{tabular}{ccccc}
\hline & PCa $(\mathbf{n}=\mathbf{1 0 5})$ & BPH $(\mathbf{n}=\mathbf{7 4})$ & Borderline $(\mathbf{n}=\mathbf{3 1})$ & HCs $(\mathbf{n}=\mathbf{1 0 4})$ \\
\hline Age $($ mean \pm SD) & $71.3 \pm 8.2$ & $66.3 \pm 7.6$ & $68.3 \pm 7.4$ & $60.1 \pm 5.8$ \\
\hline Serum PSA (ng/mL, mean \pm SD) & $33.6 \pm 107.9$ & $7.1 \pm 6.9$ & $6.0 \pm 4.2$ \\
$\leq 4$ ng/mL & 16 & 24 & 8 & 1 \\
$>4$ ng/mL & 86 & 1 & & \\
Unknown & 1 & & \\
Gleason Score (GS) & 45 & & \\
GS $=6$ & 30 & & \\
GS $=7$ & 26 & & \\
GS $\geq 8$ & 4 & & \\
Unknown &
\end{tabular}

\subsection{Highly Immunogenic Peptides}

The peptides HERV-K env ${ }_{19-37}$, HERV-K env ${ }_{109-126}$, HERV-W env $93-108$, HERV-W env $248-262$, HERV-H env $229-241$, HERV-H env $387-399$, were designed by using the Immune Epitope Database (IEBD) and synthesized at $>95 \%$ purity (LifeTein, South Plainfield, NJ, 
USA). All peptides have been resuspended in DMSO and stored in single-use aliquots at $-80{ }^{\circ} \mathrm{C}$ (Table 2).

Table 2. Aminoacidic sequences of peptides used as antigens in the ELISA assay.

\begin{tabular}{|c|c|c|c|}
\hline & Epitope Position & Epitope Sequence & UniProtKb Accession Number \\
\hline HERV-K env-su $19-37$ & $19-37$ & VWVPGPTDDRCPAKPEEEG & O42043 \\
\hline HERV-K env-su $109-126$ & $109-126$ & RPKGKTCPKEIPKGSKNT & O42043 \\
\hline HERV-W env-su'93-108 & 93-108 & NPSCPGGLGVTVCWTY & Q9UQF0 \\
\hline HERV-W env-su $248-262$ & $248-262$ & NSQCIRWVTPPTQIV & Q9UQF0 \\
\hline HERV-H env-su $229-241$ & $229-241$ & LGRHLPCISLHPW & Q9N2J8 \\
\hline HERV-H env387-399 & $387-399$ & RVIPLIPLMVGLG & Q9N2J8 \\
\hline
\end{tabular}

\subsection{Enzyme-Linked Immunosorbent Assay (ELISA)}

Indirect ELISA has been performed to evaluate the presence of a specific humoral response against highly immunogenic peptides derived from the envelope protein of HERV$\mathrm{K}, \mathrm{HERV}-\mathrm{W}$, and HERV-H. Ninety-six-well Nunc immune-plates were incubated overnight at $4{ }^{\circ} \mathrm{C}$ with $0.05 \mathrm{M}$ of carbonate-bicarbonate ( $\mathrm{pH}$ 9.5, Sigma-Aldrich, St. Louis, MO, USA), and the respective peptides at $10 \mu \mathrm{g} / \mathrm{mL}$. The plates were incubated for $1 \mathrm{~h}$ in a blocking solution with 5\% non-fat dried milk (Sigma-Aldrich, St. Louis, MO, USA) and phosphatebuffered saline (PBS), then washed twice in a solution with PBS and $0.05 \%$ Tween-20 (PBS-T). Plasma samples were added at a 1:100 concentration and incubated for $2 \mathrm{~h}$ at room temperature. After a washing step, each plate was incubated for $1 \mathrm{~h}$ with $100 \mu \mathrm{L}$ of PBS and an alkaline phosphate-conjugated goat anti-human IgG polyclonal antibody (1:1000, Sigma-Aldrich, St. Louis, MO, USA). After washing, each plate was washed in PBS-T and then incubated in milli-Q water and p-nitrophenyl phosphate (Sigma-Aldrich, St. Louis, MO, USA) for $10 \mathrm{~min}$ in a dark environment. The optical density was read at $405 \mathrm{~nm}$ using a SpectraMax Plus 384 microplate reader (Molecular Devices, Sunnyvale, CA, USA). Different negative controls were included: (1) an irrelevant peptide was present for each sample in all the experiments to verify non-specific binding; (2) one empty well (with no peptides); (3) one well where PBS was used instead of patient plasma. A competitive ELISA was performed by using HERV-K env-su ${ }_{109-126}$ and a different peptide (Annexin A2 13-37 LEGDHSTPPSAYGSVKAYTNFDAER, to which the patient was positive) in order to assess the specificity of the binding (Supplementary Figure S1). A statistically significant decrease of binding was observed only when the plasma of the positive patients was preincubated against the HERV-K-specific peptide. The results were normalized to a positive control included in all the experiments. The positive sample was previously tested for the reactivity to the selected peptides, and to an irrelevant peptide in order to verify the binding specificity. Moreover, competitive inhibition experiments were also performed, where the positive plasma before a normal ELISA was preadsorbed with the specific peptide or irrelevant peptides, in order to verify the reduction in absorbance [41,42].

\subsection{Statistical Analysis}

Data distribution was analyzed using the D'Agostino-Pearson omnibus normality test and the Shapiro-Wilk normality test. Non-parametric data were analyzed using the Mann-Whitney U test and the Kruskal-Wallis test with Dunn's multiple comparisons test to compare the antibody levels against the different HERV env-derived peptides between two or more groups, respectively. The receiver-operating characteristic (ROC) was used to select a cut-off value to assess the sample positivity, which was confirmed through Fisher's exact test. Sensitivity and specificity were chosen accordingly for all measured samples. Spearman's correlation test was performed to evaluate the correlation between the humoral response against HERV env peptides and PSA levels. The level of statistical significance 
was set up as $p<0.05$. Statistical analysis was carried out using GraphPad Prism 8.2.0 software (GraphPad Software, San Diego, CA, USA).

Supplementary Materials: The following supporting information can be downloaded at: https: / /www.mdpi.com/article/10.3390/pathogens11010095/s1, Figure S1: Competitive ELISA assay.

Author Contributions: L.A.S., supervised, designed and conceived the study, L.A.S., M.A.M., M.M. and T.S. designed the experimental design and analyzed the data statistically; M.A.M. drafted the manuscript and carried out the experiments; M.M. and T.S. recruited PCa, BPH, Borderline patients. E.R.S., M.N. and S.R. recruited Healthy Controls. M.M., T.S. and M.A.M. discussed results and approved the manuscript. L.A.S. approved the final version of the manuscript. All authors have read and agreed to the published version of the manuscript.

Funding: This work was supported by Fondazione di Sardegna 2017 to Sechi LA, and UNISS fondi ricerca 2020 to Sechi LA.

Institutional Review Board Statement: The study was conducted in accordance with th Declaration of Helsinki and approved by the Institutional Review Board.

Informed Consent Statement: Informed Consent statement was signed by all patients and Healthy Controls.

Conflicts of Interest: The authors declare no conflict of interest.

\section{References}

1. Pernar, C.H.; Ebot, E.M.; Wilson, K.M.; Mucci, L.A. The Epidemiology of Prostate Cancer. Cold Spring Harb. Perspect. Med. 2018, 8, a030361. [CrossRef]

2. Matsushita, M.; Fujita, K. Influence of Diet and Nutrition on Prostate Cancer. Int. J. Mol. Sci. 2020, 21, 1447. [CrossRef]

3. Perdana, N.R.; Mochtar, C.A.; Umbas, R.; Hamid, A.R.A. The Risk Factors of Prostate Cancer and Its Prevention: A Literature Review. Acta Med. Indones. 2016, 48, 228-238. [PubMed]

4. Rani, A.; Dasgupta, P.; Murphy, J.J. Prostate Cancer: The Role of Inflammation and Chemokines. Am. J. Pathol. 2019, 189, 2119-2137. [CrossRef] [PubMed]

5. Nakai, Y.; Nonomura, N. Inflammation and prostate carcinogenesis. Int. J. Urol. 2013, 20, 150-160. [CrossRef]

6. Saini, S. PSA and beyond: Alternative prostate cancer biomarkers. Cell. Oncol. 2016, 39, 97-106. [CrossRef] [PubMed]

7. Geis, F.K.; Goff, S.P. Silencing and Transcriptional Regulation of Endogenous Retroviruses: An Overview. Viruses 2020, 12, 884. [CrossRef] [PubMed]

8. Grandi, N.; Tramontano, E. Human endogenous retroviruses are ancient acquired elements still shaping innate immune responses. Front. Immunol. 2018, 9, 2039. [CrossRef]

9. Grandi, N.; Tramontano, E. HERV envelope proteins: Physiological role and pathogenic potential in cancer and autoimmunity. Front. Microbiol. 2018, 9, 462. [CrossRef] [PubMed]

10. Downey, R.F.; Sullivan, F.J.; Wang-Johanning, F.; Ambs, S.; Giles, F.J.; Glynn, S.A. Human endogenous retrovirus K and cancer: Innocent bystander or tumorigenic accomplice? Int. J. Cancer 2015, 137, 1249-1257. [CrossRef] [PubMed]

11. Xue, B.; Sechi, L.; Kelvin, D. Human Endogenous Retrovirus K (HML-2) in Health and Disease. Front. Microbiol. 2020, 11, 1690. [CrossRef]

12. Turner, G.; Barbulescu, M.; Su, M.; Jensen-Seaman, M.I.; Kidd, K.K.; Lenz, J. Insertional polymorphisms of full-length endogenous retroviruses in humans. Curr. Biol. 2001, 11, 1531-1535. [CrossRef]

13. Salavatiha, Z.; Soleimani-Jelodar, R.; Jalilvand, S. The role of endogenous retroviruses-K in human cancer. Rev. Med. Virol. 2020, 30, 1-13. [CrossRef]

14. Armbruester, V.; Sauter, M.; Krautkraemer, E.; Meese, E.; Kleiman, A.; Best, B.; Roemer, K.; Mueller-Lantzsch, N. A novel gene from the human endogenous retrovirus K expressed in transformed cells. Clin. Cancer Res. 2002, 8, 1800-1807.

15. Lower, R.; Tonjes, R.; Korbmacher, C.; Kurth, R.; Lower, J. Identification of a Rev-Related Protein by Analysis of Spliced Transcripts of the Human Endogenous. Microbiology 1995, 69, 141-149.

16. Huang, W.; Chen, T.; Meng, Z.; Gan, Y.; Wang, X.; Xu, F.; Gu, Y.; Xu, X.; Tang, J.; Zhou, H.; et al. The viral oncogene Np9 acts as a critical molecular switch for co-activating $\beta$-catenin, ERK, Akt and Notch1 and promoting the growth of human leukemia stem/progenitor cells. Leukemia 2013, 27, 1469-1478. [CrossRef]

17. Denne, M.; Sauter, M.; Armbruester, V.; Licht, J.D.; Roemer, K.; Mueller-lantzsch, N. Physical and Functional Interactions of Human Endogenous Retrovirus Proteins Np9 and Rec with the Promyelocytic Leukemia Zinc Finger Protein. J. Virol. 2007, 81, 5607-5616. [CrossRef]

18. Schmitt, K.; Heyne, K.; Roemer, K.; Meese, E.; Mayer, J. HERV-K(HML-2) Rec and Np9 transcripts not restricted to disease but present in many normal human tissues. Mob. DNA 2015, 6, 4. [CrossRef]

19. Xue, B.; Zeng, T.; Jia, L.; Yang, D.; Lin, S.L.; Sechi, L.A.; Kelvin, D.J. Identification of the distribution of human endogenous retroviruses K (HML-2) by PCR-based target enrichment sequencing. Retrovirology 2020, 17, 10. [CrossRef] 
20. Hahn, S.; Ugurel, S.; Hanschmann, K.M.; Strobel, H.; Tondera, C.; Schadendorf, D.; Löwer, J.; Löwer, R. Serological response to human endogenous retrovirus $\mathrm{K}$ in melanoma patients correlates with survival probability. AIDS Res. Hum. Retrovir. 2008, 24, 717-723. [CrossRef]

21. Schmitt, K.; Reichrath, J.; Roesch, A.; Meese, E.; Mayer, J. Transcriptional profiling of human endogenous retrovirus group HERV-K(HML-2) loci in melanoma. Genome Biol. Evol. 2013, 5, 307-328. [CrossRef]

22. Wang-Johanning, F.; Radvanyi, L.; Rycaj, K.; Plummer, J.B.; Yan, P.; Sastry, K.J.; Piyathilake, C.J.; Hunt, K.K.; Johanning, G.L. Human endogenous retrovirus $\mathrm{K}$ triggers an antigen-specific immune response in breast cancer patients. Cancer Res. 2008, 68, 5869-5877. [CrossRef]

23. Chan, S.M.; Sapir, T.; Park, S.; Id, R.; Contreras-galindo, R.; Reiner, O.; Id, D.M.M. The HERV-K accessory protein Np9 controls viability and migration of teratocarcinoma cells. PLOS ONE 2019, 14, e0212970. [CrossRef]

24. Depil, S.; Roche, C.; Dussart, P.; Prin, L. Expression of a human endogenous retrovirus, HERV-K, in the blood cells of leukemia patients. Leukemia 2002, 16, 254-259. [CrossRef]

25. Reis, B.S.; Jungbluth, A.A.; Frosina, D.; Holz, M.; Ritter, E.; Nakayama, E.; Ishida, T.; Obata, Y.; Carver, B.; Scher, H.; et al. Prostate cancer progression correlates with increased humoral immune response to a human endogenous retrovirus GAG protein. Clin. Cancer Res. 2013, 19, 6112-6125. [CrossRef]

26. Wallace, T.A.; Downey, R.F.; Seufert, C.J.; Schetter, A.; Dorsey, T.H.; Johnson, C.A.; Goldman, R.; Loffredo, C.A.; Yan, P.; Sullivan, F.J.; et al. Elevated HERV-K mRNA expression in PBMC is associated with a prostate cancer diagnosis particularly in older men and smokers. Carcinogenesis 2014, 35, 2074-2083. [CrossRef]

27. Patzke, S.; Lindeskog, M.; Munthe, E.; Aasheim, H.C. Characterization of a novel human endogenous retrovirus, HERV-H/F, expressed in human leukemia cell lines. Virology 2002, 303, 164-173. [CrossRef]

28. Mullins, C.S.; Linnebacher, M. Endogenous retrovirus sequences as a novel class of tumor-specific antigens: An example of HERV-H env encoding strong CTL epitopes. Cancer Immunol. Immunother. 2012, 61, 1093-1100. [CrossRef]

29. Gimenez, J.; Montgiraud, C.; Pichon, J.P.; Bonnaud, B.; Arsac, M.; Ruel, K.; Bouton, O.; Mallet, F. Custom human endogenous retroviruses dedicated microarray identifies self-induced HERV-W family elements reactivated in testicular cancer upon methylation control. Nucleic Acids Res. 2010, 38, 2229-2246. [CrossRef]

30. Küry, P.; Nath, A.; Créange, A.; Dolei, A.; Marche, P.; Gold, J.; Giovannoni, G.; Hartung, H.P.; Perron, H. Human Endogenous Retroviruses in Neurological Diseases. Trends Mol. Med. 2018, 24, 379-394. [CrossRef]

31. Arru, G.; Mameli, G.; Deiana, G.A.; Rassu, A.L.; Piredda, R.; Sechi, E.; Caggiu, E.; Bo, M.; Nako, E.; Urso, D.; et al. Humoral immunity response to human endogenous retroviruses $\mathrm{K} / \mathrm{W}$ differentiates between amyotrophic lateral sclerosis and other neurological diseases. Eur. J. Neurol. 2018, 25, 1076-e84. [CrossRef]

32. Mameli, G.; Erre, G.L.; Caggiu, E.; Mura, S.; Cossu, D.; Bo, M.; Cadoni, M.L.; Piras, A.; Mundula, N.; Colombo, E.; et al. Identification of a HERV-K env surface peptide highly recognized in Rheumatoid Arthritis (RA) patients: A cross-sectional case-control study. Clin. Exp. Immunol. 2017, 189, 127-131. [CrossRef] [PubMed]

33. Kewitz, S.; Staege, M.S. Expression and regulation of the endogenous retrovirus 3 in Hodgkin's lymphoma cells. Front. Oncol. 2013, 3, 179. [CrossRef]

34. Ishida, T.; Obata, Y.; Ohara, N.; Matsushita, H.; Sato, S.; Uenaka, A.; Saika, T.; Miyamura, T.; Chayama, K.; Nakamura, Y.; et al. Identification of the HERV-K gag antigen in prostate cancer by SEREX using autologous patient serum and its immunogenicity. Cancer Immun. 2008, 8, 1-10.

35. Benešová, M.; Trejbalová, K.; Kovářová, D.; Vernerová, Z.; Hron, T.; Kučerová, D.; Hejnar, J. DNA hypomethylation and aberrant expression of the human endogenous retrovirus ERVWE1/syncytin-1 in seminomas. Retrovirology 2017, 14, 20. [CrossRef] [PubMed]

36. Barth, M.; Gröger, V.; Cynis, H.; Staege, M.S. Identification of human endogenous retrovirus transcripts in Hodgkin Lymphoma cells. Mol. Biol. Rep. 2019, 46, 1885-1893. [CrossRef] [PubMed]

37. Lemaître, C.; Tsang, J.; Bireau, C.; Heidmann, T.; Dewannieux, M. A human endogenous retrovirus-derived gene that can contribute to oncogenesis by activating the ERK pathway and inducing migration and invasion. PLoS Pathog. 2017, 13, e1006451. [CrossRef] [PubMed]

38. Tachedjian, G. HERV-K Gag RNA and Protein Levels Are Elevated Prostate Cancer. Viruse 2021, 13, 449.

39. Kudo-Saito, C.; Yura, M.; Yamamoto, R.; Kawakami, Y. Induction of immunoregulatory CD271+ cells by metastatic tumor cells that express human endogenous retrovirus H. Cancer Res. 2014, 74, 1361-1370. [CrossRef] [PubMed]

40. Pérot, P.; Mullins, C.S.; Naville, M.; Bressan, C.; Hühns, M.; Gock, M.; Kühn, F.; Volff, J.N.; Trillet-Lenoir, V.; Linnebacher, M.; et al. Expression of young HERV-H loci in the course of colorectal carcinoma and correlation with molecular subtypes. Oncotarget 2015, 6, 40095-40111. [CrossRef]

41. Simula, E.R.; Manca, M.A.; Jasemi, S.; Uzzau, S.; Rubino, S.; Manchia, P.; Bitti, A.; Palermo, M.; Sechi, L.A. HCoV-NL63 and SARS-CoV-2 Share Recognized Epitopes by the Humoral Response in Sera of People Collected Pre- and during CoV-2 Pandemic. Microorganisms 2020, 8, 1993. [CrossRef] [PubMed]

42. Masala, S.; Paccagnini, D.; Cossu, D.; Brezar, V.; Pacifico, A.; Ahmed, N.; Mallone, R.; Sechi, L.A. Antibodies Recognizing Mycobacterium avium paratuberculosis Epitopes Cross-React with the Beta-Cell Antigen ZnT8 in Sardinian Type 1 Diabetic Patients. PLoS ONE 2011, 6, e26931. [CrossRef] [PubMed] 